Felices de la Fuente, M.M. \& Chaparro Sainz, A. (2021). Representaciones del currículo social en el futuro profesorado de Educación Infantil. Una investigación cualitativa. Revista Electrónica Interuniversitaria de Formación del Profesorado, 24(1), 145-161.

DOI: https://doi.org/10.6018/reifop.394791

\title{
Representaciones del currículo social en el futuro profesorado de Educación Infantil. Una investigación cualitativa*
}

\author{
María del Mar Felices de la Fuente ${ }^{1}$, Álvaro Chaparro Sainz ${ }^{2}$ \\ 'Universidad de Almería, ${ }^{2}$ Universidad de Málaga
}

\section{Resumen}

Esta investigación tiene por objeto evaluar las competencias curriculares del alumnado del Grado en Educación Infantil, tras cursar las asignaturas de Didáctica de las Ciencias Sociales. Para ello, nos centramos en examinar dos indicadores principales: si emplea de forma apropiada el principio de globalidad que debe presidir los procesos de enseñanza y aprendizaje de esta etapa, y si reconoce e identifica el contenido social curricular. Siguiendo una metodología cualitativa y empleando los programas informáticos Nvivo 11 y Pajek 5.07, se han analizado contenidos textuales generados por 224 alumnos y alumnas, procedentes de ISEN (Universidad de Murcia) y de la Universidad de Málaga, a quienes se les pidió que, a partir de la elección de un contenido social del currículo, propusieran una situación de aprendizaje integrado. Los resultados nos muestran dificultades tanto en la identificación de las temáticas específicamente sociales, como en la conexión entre contenidos de las distintas áreas del currículo. En consecuencia, se revela necesario un tratamiento más exhaustivo de estos aspectos desde el ámbito de la Didáctica de las Ciencias Sociales, que implique un mejor conocimiento y delimitación de lo "social" por parte del futuro profesorado de Educación Infantil.

\section{Palabras clave}

Educación Infantil; formación inicial de profesorado; competencias curriculares específicas; didáctica de las Ciencias Sociales.

\footnotetext{
Contacto:

Álvaro Chaparro Sainz. Departamento de Didáctica de la Matemática, de las Ciencias Sociales y de las Ciencias Experimentales Facultad de Ciencias de la Educación. Universidad de Málaga. alvaro.chaparro@uma.es

* Este trabajo es resultado del proyecto de investigación "Conceptos metodológicos y métodos activos de aprendizaje para la mejora de las competencias docentes del profesorado" (PGC2018-094491-B-C33), subvencionado por el Ministerio de Ciencia, Innovación y Universidades de España, cofinanciado con fondos FEDER.
} 


\title{
Representations of social curriculum in pre-service preschool teachers. A qualitative research.
}

\begin{abstract}
The objective of this research is to evaluate the curricular competences of the students of the Degree in Preschool Education, after having completed the subject of Social Sciences Education. In order to achieve this purpose, we focus on examining two indicators: if the students adequately use the principle of globality that should preside over the teaching and learning processes of the preschool stage; and if they recognizes and identifies the curricular social content. For this, from a qualitative methodological approach, we have used the software Nvivo 11 and Pajek64 5.07, to analyze the textual contents generated by 224 students from ISEN (University of Murcia) and the University of Málaga. All of them were asked to choose a social content of the curriculum and to propose an integrated learning situation based on it. The research results show us difficulties in the identification of specifically social issues, and in the connection between contents of the different areas of the curriculum. Consequently, it is evident that a more exhaustive treatment of these aspects is necessary from the scope of the Social Sciences Education, which implies a better knowledge and delimitation of the social content by the future preschool teachers.
\end{abstract}

\section{Key words}

Preschool; initial teacher training; teacher qualifications; social sciences education.

\section{Introducción}

La presente investigación tiene por objeto evaluar si el alumnado del Grado en Educación Infantil adquiere las competencias previstas en esta titulación y es capaz de ponerlas en práctica. De entre las competencias recogidas en la ORDEN ECI/3854/2007, de 27 de diciembre, por la que se establecen los requisitos para la verificación de los títulos universitarios oficiales que habilitan para ejercer la profesión de maestro/a de Educación Infantil, nos interesa profundizar en el alcance de dos de ellas: "1. Conocer los objetivos, contenidos curriculares y criterios de evaluación de la Educación Infantil” y "2. Promover y facilitar los aprendizajes en la primera infancia, desde una perspectiva globalizadora e integradora de las diferentes dimensiones cognitiva, emocional, psicomotora y volitiva". Hemos seleccionado ambas competencias porque se corresponden con nuestros objetivos de investigación: determinar si el futuro profesorado de Educación Infantil identifica el contenido social curricular y si puede relacionarlo con otros contenidos de distintas áreas, aplicando el principio de globalidad que debe vertebrar todos los procesos de enseñanza y aprendizaje de esta etapa.

Nos interesa, por tanto, profundizar en el nivel de adquisición de competencias curriculares específicas del maestro, tal y como las definió Rosales López (2013: 74-75), quien estableció una clasificación de competencias basada en: competencias para estimular los aprendizajes propios de las etapas; competencias vinculadas son los contextos de intervención; y las mencionadas competencias curriculares específicas. Estas últimas abrazan aquellas que están relacionadas con aspectos como la planificación de la enseñanza, la selección y organización de contenidos, la utilización de los métodos, técnicas y recursos adaptados al contexto educativo, y la evaluación de los aprendizajes y de la propia docencia, lo que 
contempla un fuerte vínculo con la reflexión, la investigación y la innovación en la práctica docente (Rosales, 2013: 80).

En este trabajo, nos centramos concretamente en las competencias adquiridas por el alumnado universitario para seleccionar y organizar los contenidos, pues teniendo en cuenta que el maestro/a de infantil es un profesional generalista, responsable del aprendizaje de conocimientos de distintos ámbitos, es preciso que sea competente a la hora de seleccionarlos y diferenciarlos. Asimismo, queremos evaluar la capacidad de integración de contenidos, en torno a centros de interés del propio alumnado, atendiendo a los principios de motivación y globalización de los aprendizajes (Rosales, 2013: 81).

Esta investigación se ha contextualizado en el campo de estudio de la Didáctica de las Ciencias Sociales, pues como docentes de este ámbito, nos inquieta el escaso espacio que ocupan las Ciencias Sociales en el currículo, frente a otros aprendizajes, así como las dificultades que el alumnado universitario en formación muestra para identificar el conocimiento social. En este sentido, son diversos los trabajos que han puesto de relieve la reducida e incluso anecdótica presencia de las Ciencias Sociales en esta etapa, señalando incluso que su aparición es mayormente monotemática, de carácter actitudinal (Cuenca, 2008), y principalmente enfocada hacia la socialización del alumnado en ámbitos como la familia, la escuela o la localidad (Miralles y Molina, 2011). Esta situación obstaculiza una adecuada integración de conceptos como el espacio y el tiempo o, incluso, la dimensión cultural, circunstancia muy relacionada con la influencia en el currículo de los estudios de Piaget y otros investigadores que consideran difícil para el niño/a de estas edades introducirse en el conocimiento del tiempo histórico o del espacio lejano o no vivenciado.

Pese al influjo de estas teorías, lo cierto es que, desde hace décadas, las investigaciones en este ámbito han superado estas concepciones demostrando que el aprendizaje de la geografía y el tiempo histórico no depende tanto del desarrollo cognitivo del niño, como de los contenidos, estrategias metodológicas y recursos que se empleen (Calvani, 1988; Cuenca y Domínguez, 2000; Tonda, 2001; Cooper, 2002; Trepat, 2011; Miralles y Rivero, 2012; o Aranda, 2016, entre otros), así como del nivel de experimentación, el entorno social y los factores ambientales (Comes, 2002; Rivero y Gil, 2011; Aranda, 2016).

Creemos que una adecuada formación del profesorado en el tratamiento de lo "social" puede favorecer procesos de enseñanza y aprendizaje que ayuden al desarrollo de competencias sociales y ciudadanas. Por ello, nos preocupa conocer en qué medida estamos favoreciendo un adecuado desarrollo profesional de los futuros maestros (Alsina, 2013). Desde un enfoque reflexivo-crítico, esta investigación nos permitirá detectar necesidades y mejorar nuestras prácticas docentes.

\section{Método}

\section{Objetivos de la investigación}

El objetivo principal de esta investigación es evaluar si el alumnado ha alcanzado las competencias previstas en su formación inicial, centrándonos concretamente en la competencia específica de conocimiento del currículo social de la etapa de Educación Infantil. Este objetivo se compone a su vez de dos subobjetivos:

1. Evaluar la capacidad del alumnado para plantear aprendizajes desde una perspectiva globalizadora e integrada de las diferentes áreas que recoge el currículo. 
2. Analizar la capacidad del alumnado para identificar el conocimiento social curricular de Educación Infantil.

\section{Participantes}

En este estudio han participado 224 estudiantes $(n=224)$ del Grado en Educación Infantil, matriculados en tercer y cuarto año durante los cursos académicos 2016/2017 y 2017/2018, que cursaron la asignatura de Didáctica de las Ciencias Sociales. La muestra se compone de 216 mujeres ( $96,4 \%$ ) y 8 hombres (3,6\%), lo que supone un importante desequilibrio por género, con una amplia mayoría de alumnas frente a un pequeño porcentaje de alumnos. El porcentaje de participación de cada universidad es similar, pues 109 estudiantes $(n=109)$ proceden de ISEN (centro universitario adscrito a la Universidad de Murcia), representando un $48,7 \%$ de la muestra; y $115(n=115)$ son de la Universidad de Málaga, lo que supone un $51,3 \%$ del total.

El tipo de muestreo realizado ha sido no probabilístico y de conveniencia, ya que la selección del alumnado participante en el estudio viene determinada por la accesibilidad a los sujetos y por su adecuación a los objetivos contemplados en la investigación (Sáez López, 2017: 66).

\section{Diseño y procedimiento}

Esta investigación se sitúa en el paradigma de la investigación constructivista o cualitativa (teoría interpretativa), que pretende describir para comprender e interpretar qué sucede en un contexto determinado (Bisquerra, 2004; Cohen y Manion, 2002). En consecuencia, la metodología utilizada en este estudio ha sido de carácter cualitativo y de corte descriptivoexploratorio.

Para la recogida inicial de información se empleó la red social Pinterest donde, tras crear un tablero público titulado "Conocimiento del entorno", al que se dio acceso a todos los participantes, se les solicitó que subieran a la citada plataforma, al menos, una imagen representativa de un contenido social. Además, se les pidió que explicaran, a través de un texto, cómo abordar ese contenido desde una perspectiva globalizada, vinculándolo con otras áreas y contenidos del currículo. De este modo, pudimos recoger producciones textuales del alumnado, en las que se reflejasen sus aprendizajes y concepciones acerca del conocimiento social y el principio de integración de contenidos. La elección de la red social Pinterest como instrumento para la recogida de información se justifica por dos motivos: introducir nuevos recursos en las prácticas docentes para mostrar a los estudiantes sus posibilidades didácticas; y desarrollar sus competencias digitales y la toma de decisiones pedagógicas relacionadas con el uso de las nuevas tecnologías (Tárraga-Mínguez et al., 2017).

La incorporación total de imágenes a Pinterest, junto con sus respectivos textos explicativos, ha ascendido a 748 producciones (3,3 por persona de media). Dada la naturaleza de la población participante, todos ellos estudiantes del Grado en Educación Infantil, se vio conveniente establecer una muestra para su análisis. Para la selección de la muestra de imágenes, se tuvo en cuenta la primera fotografía y texto incorporado por cada uno de los 224 participantes, a fin de favorecer la representación de todos ellos.

Para analizar las 224 producciones textuales seleccionadas, se diseñó ad hoc una ficha de análisis para el registro de datos (Piñuel, 2002: 23-24) donde se vació toda la información disponible en la plataforma para analizarla y categorizarla. 
En la sistematización de datos hemos empleado el análisis descriptivo e inferencial de contenido manifiesto propuesto por Piñuel (2002), que lleva consigo una serie de procedimientos interpretativos de productos comunicativos, en este caso, producciones del alumnado, que han sido tratados mediante técnicas cualitativas, basadas en la combinación de categorías. En este proceso de análisis de contenido, de carácter verificativo y explicativo, se han tenido en cuenta también las fases de análisis de contenido propuestas por López, Domínguez, Álvarez y Jaén (2011). Siguiendo sus orientaciones, dividimos el proceso de análisis de datos en dos fases: fase de reducción de datos y fase de interpretación de los mismos. En la primera fase, hemos categorizado y codificado la información, con ayuda del programa Nvivo 11, que se utiliza para el análisis cualitativo de la muestra, así como para el análisis del discurso (Blasco y Mengual, 2010: 77). En nuestro caso, nos ha servido para codificar la información, creando nodos vinculados a estructuras textuales concretas (Valdemoros, Ponce de León y Sanz, 2011). Para el codificado de los textos hemos definido las unidades de información o nodos siguiendo el criterio temático (temas) que estaba presente en ellas, adoptando un proceso de categorización inductivo (Gil y Perera, 2001).

Para atender a los dos objetivos de investigación señalados más arriba, se han establecido dos niveles de codificación. Por un lado, se ha creado una categoría para cada uno de los once bloques de contenidos que conforman las tres áreas existentes en el currículo de Educación Infantil (Tabla 1), pudiendo atribuir a cada texto, una o varias categorías en función de su contenido. Estas codificaciones nos han servido para establecer el primer nivel de análisis de resultados, que se corresponde con el primer objetivo de la investigación: evaluar la capacidad del alumnado para plantear aprendizajes desde una perspectiva globalizadora de las diferentes áreas que recoge el currículo.

Tabla 1.

Codificación de los bloques de contenidos del currículo de Educación Infantil ${ }^{*}$

\begin{tabular}{|c|c|c|}
\hline Área del currículo & Bloque de contenido & Codificación \\
\hline \multirow{4}{*}{$\begin{array}{l}\text { Conocimiento de } \\
\text { sí mismo y } \\
\text { autonomía } \\
\text { personal }\end{array}$} & Bloque 1. El cuerpo y la propia imagen & A1.B1 \\
\hline & Bloque 2. Juego y movimiento & A1.B2 \\
\hline & Bloque 3. La actividad y la vida cotidiana & A1.B3 \\
\hline & Bloque 4. El cuidado personal y la salud & A1.B4 \\
\hline $\begin{array}{l}\text { 2. Conocimiento del } \\
\text { entorno }\end{array}$ & $\begin{array}{l}\text { Bloque } 1 . \text { Medio físico: elementos, } \\
\text { relaciones y medida }\end{array}$ & $\mathrm{A} 2 . \mathrm{B} 1$ \\
\hline & Bloque 2. Acercamiento a la naturaleza & $A 2 . B 2$ \\
\hline & Bloque 3. Cultura y vida en sociedad & A2.B3 \\
\hline 3. Lenguajes: & Bloque 1. Lenguaje verbal & A3.B1 \\
\hline $\begin{array}{l}\text { comunicación y } \\
\text { representación }\end{array}$ & $\begin{array}{l}\text { Bloque 2. Lenguaje audiovisual y } \\
\text { tecnologías de la información y la } \\
\text { comunicación }\end{array}$ & A3.B2 \\
\hline & Bloque 3. Lenguaje artístico & A3.B3 \\
\hline & Bloque 4 . Lenguaje corporal & A3.B4 \\
\hline
\end{tabular}

También se han creado categorías para las diferentes temáticas libres que, a voluntad del alumnado participante, fueron progresivamente apareciendo en las descripciones de las

\footnotetext{
* Real Decreto, 1630/2006, de 29 de diciembre, por el que se establecen las enseñanzas mínimas del segundo ciclo de Educación Infantil. BOE, núm. 4, publicado el 4 de enero de 2007.
} 
imágenes (Tabla 2). Analizadas sistemáticamente las producciones seleccionadas de los participantes, se definieron las categorías que ascienden a un total de 21. Este proceso nos ha servido para establecer el segundo nivel de análisis de resultados, que se corresponde a su vez con el segundo objetivo de la investigación: analizar la capacidad del alumnado para reconocer y delimitar el conocimiento social en el currículo de Educación Infantil.

Tabla 2.

Categorización de tópicos presentes en las producciones del alumnado.

\begin{tabular}{|c|c|}
\hline Categorías & Descripción de la categoría \\
\hline Alimentación & Alimentos y gastronomía tradicional. \\
\hline Astronomía & $\begin{array}{l}\text { Estrellas, planetas, sistema solar, o elementos relacionados con el } \\
\text { universo. }\end{array}$ \\
\hline Currículo & Principios o elementos propios del currículo de Educación Infantil. \\
\hline $\begin{array}{l}\text { Actividades } \\
\text { socioeconómicas }\end{array}$ & $\begin{array}{l}\text { Actividades sociales y de intercambio, de carácter principalmente } \\
\text { económico. }\end{array}$ \\
\hline Educación vial & $\begin{array}{l}\text { Normas de circulación, usos de vías públicas, señales de tráfico, } \\
\text { comportamientos cívicos, etc. }\end{array}$ \\
\hline Familia & $\begin{array}{l}\text { Contenidos vinculados al ámbito familiar, como parientes, lazos } \\
\text { familiares, emociones, contexto familiar, etc. }\end{array}$ \\
\hline Género-igualdad & Coeducación y la igualdad de género. \\
\hline Historia & Manifestaciones o hechos históricos, excluyendo lo patrimonial. \\
\hline Medios de transporte & Referencias a transportes. \\
\hline Metodologías & Recursos, estrategias, formas de enseñar, etc. \\
\hline $\mathrm{ABN}$ & $\begin{array}{l}\text { Método Abierto Basado en Números o método de cálculo ABN, del } \\
\text { ámbito de las matemáticas. }\end{array}$ \\
\hline Entorno & $\begin{array}{l}\text { Concepto “entorno”, elementos del entorno social, natural o cultural, } \\
\text { preferiblemente próximo. }\end{array}$ \\
\hline Salidas didácticas & $\begin{array}{l}\text { Salidas didácticas e itinerarios, propuestas por los estudiantes, como } \\
\text { recursos para la enseñanza y el aprendizaje. }\end{array}$ \\
\hline Música & $\begin{array}{l}\text { Elementos musicales, como instrumentos, museos de la música, } \\
\text { canciones, etc. }\end{array}$ \\
\hline Naturaleza & $\begin{array}{l}\text { Elementos de la naturaleza, como flora fauna, espacios naturales, } \\
\text { parques, etc. }\end{array}$ \\
\hline Paisaje & Paisajes o elementos paisajísticos, no exclusivamente naturales. \\
\hline Patrimonio & $\begin{array}{l}\text { Patrimonio, tanto material como inmaterial, de carácter natural, } \\
\text { industrial, histórico, artístico, etc. }\end{array}$ \\
\hline Profesiones & Profesiones antiguas y contemporáneas. \\
\hline Pueblo-ciudad & Medio urbano y medio rural. \\
\hline Reciclaje & $\begin{array}{l}\text { Prácticas de concienciación sobre la conservación del medio } \\
\text { ambiente y el tratamiento de residuos. }\end{array}$ \\
\hline Seres vivos & $\begin{array}{l}\text { Seres vivos, relativos tanto a flora como a fauna, y a la necesidad de } \\
\text { concienciarnos sobre su conservación. }\end{array}$ \\
\hline
\end{tabular}

Para la conveniente visualización de las relaciones que se establecen entre los datos analizados, se ha utilizado el programa de análisis de redes sociales Pajek 5.07, entendiendo redes sociales como conexiones relacionales entre elementos, no desde el punto de vista de comunidades virtuales e interactivas. A partir del trabajo con este software ha sido posible representar gráficamente el conjunto de relaciones o interacciones producidas como consecuencia de la codificación de la muestra. Para la representación, hemos optado por el algoritmo Kamada Kawai que posibilita la visualización de los nodos (categorías codificadas) calibrados en función del peso que cada uno de ellos posee en el interior de la red. 


\section{Resultados}

Teniendo en cuenta los dos niveles de análisis mencionados, los resultados relativos al primer nivel, referente a la capacidad de aplicación del principio de globalidad del alumnado del Grado en Educación Infantil, son los que se muestran en la Tabla 3.

Tabla 3.

Presencia del principio de globalidad en las producciones del alumnado

\begin{tabular}{|c|c|c|c|c|}
\hline Área & Bloque de contenidos & Referencias & Total & $\%$ \\
\hline \multirow{4}{*}{$\begin{array}{l}\text { Conocimiento de } \\
\text { sí mismo y } \\
\text { autonomía } \\
\text { personal }\end{array}$} & (A1.B1) El cuerpo y la propia imagen & 3 & \multirow{4}{*}{59} & \multirow{4}{*}{$21,77 \%$} \\
\hline & (A1.B2) Juego y movimiento & 3 & & \\
\hline & (A1.B3) La actividad y la vida cotidiana & 41 & & \\
\hline & (A1.B4) El cuidado personal y la salud & 12 & & \\
\hline \multirow{3}{*}{$\begin{array}{l}\text { Conocimiento } \\
\text { del entorno }\end{array}$} & $\begin{array}{l}\text { (A2.B1) Medio físico: Elementos, } \\
\text { relaciones y medidas }\end{array}$ & 8 & \multirow[t]{3}{*}{175} & \multirow[t]{3}{*}{$64,57 \%$} \\
\hline & (A2.B2) Acercamiento a la naturaleza & 88 & & \\
\hline & (A2.B3) Cultura y vida en sociedad & 79 & & \\
\hline \multirow{4}{*}{$\begin{array}{l}\text { Lenguajes: } \\
\text { comunicación y } \\
\text { representación }\end{array}$} & (A3.B1) Lenguaje verbal & 9 & \multirow{4}{*}{37} & \multirow{4}{*}{$13,65 \%$} \\
\hline & $\begin{array}{l}\text { (A3.B2) Lenguaje audiovisual y } \\
\text { tecnologías de la información y de la } \\
\text { comunicación }\end{array}$ & 5 & & \\
\hline & (A3.B3) Lenguaje artístico & 12 & & \\
\hline & (A3.B4) Lenguaje corporal & 11 & & \\
\hline \multicolumn{2}{|c|}{ Total de referencias o nodos } & & 271 & $100,00 \%$ \\
\hline
\end{tabular}

En una primera aproximación a los datos, observamos cómo el alumnado ha incluido en sus textos 96 referencias -de las 271 registradas- a contenidos de bloques distintos a los que conforman el área 2. "Conocimiento del entorno", donde se engloba el contenido social, concretamente en su bloque 3. Estas referencias se distribuyen en 59 para el área 1. "Conocimiento de sí mismo y autonomía personal" y 37 para el área 3. "Lenguajes: comunicación y representación". La codificación de estas categorías, y su posterior análisis, ha permitido observar cómo el principio de globalidad con otras áreas se registra en un $35,42 \%$ de los casos, lo que puede interpretarse como un valor aceptable, aunque insuficiente, si tenemos en cuenta la importancia de la integración de contenidos en esta etapa.

Como era de esperar, el mayor número de referencias corresponden a contenidos de los nodos A2.B2 y A2.B3, del área 2. "Conocimiento del entorno". Este hecho se explica porque ambos bloques presentan contenidos muy relacionados entre sí, vinculados con el entorno natural, social y cultural del niño/a. Cabe destacar, sin embargo, las escasas referencias al nodo A2.B1, incluido en la misma área, pero apenas mencionado. En este sentido, el estudio refleja las dificultades del alumnado para vincular los contenidos de este bloque, de carácter lógico-matemático, con los contenidos sociales, pese a que el aprendizaje del espacio y el tiempo posibilite el desarrollo del pensamiento matemático y viceversa, lo que nos muestra ciertas carencias del estudiantado para aplicar la capacidad globalizadora requerida. 
Además, resulta reseñable la mayor mención al medio natural ( 88 nodos), correspondiente al bloque $A 2 . B 2$, respecto de los elementos de la categoría $A 2 . B 3$, donde se recogen, como ya hemos señalado, los contenidos propios de Ciencias Sociales (79 nodos). Esta circunstancia llama la atención si tenemos en cuenta que, en las instrucciones dadas al alumnado, se les pidió que las imágenes tuvieran como referente contenidos sociales, y que, en consecuencia, las explicaciones versaran sobre cómo abordarlos desde una perspectiva integradora. El mayor volumen de referencias a contenidos del medio natural en detrimento del social, pese a ser leve, nos alerta sobre problemas para identificar contenidos relativos a la relación del ser humano en y con el medio que le rodea. Se infieren, por tanto, obstáculos para que el alumnado discierna correctamente entre medio social (grupos sociales, comunidad, señas de identidad, actividades sociales y culturales, etc.) y medio natural.

Por otro lado, observamos que los estudiantes han conectado los contenidos sociales, principalmente, con la categoría A1.B3 (41 nodos) del área 1. "Conocimiento de sí mismo y autonomía personal”. Un vínculo que podríamos considerar lógico, dado que en este bloque encontramos contenidos que se podrían relacionar con el contenido social por el amplio enfoque socializador que se le otorga en el currículo. Es un bloque que integra contenidos sobre actividades, valores y normas que regulan nuestras actuaciones, así como la interacción, colaboración y actitud positiva para establecer vínculos con las personas. La conexión con este bloque, aunque predecible por considerarse próxima a los conocimientos sociales, resulta altamente positiva.

Mucho más reducidas han sido las conexiones entre contenidos del ámbito social y contenidos de bloques como, por ejemplo, el nodo A1.B1 y A1.B2 del área 1. "Conocimiento de sí mismo y autonomía personal", relativos al cuerpo y la propia imagen, y el juego y movimiento. Ambos bloques han sido, con diferencia, los menos referenciados, siendo solo 3 las veces que se han nombrado. Destacable además es la escasa relación que se aprecia entre el conocimiento social y el área 3 del currículo, dedicada al Lenguaje. Más llamativa aún resulta esta desconexión con el nodo A3.B2, sobre todo, si tenemos en cuenta la importancia que se da en la actualidad al lenguaje audiovisual y a las nuevas tecnologías.

A fin de concretar visualmente los resultados de este primer nivel de análisis y de determinar el nivel de aplicación del principio globalizador en los textos analizados, incluimos el Gráfico 1, realizado con el software Pajek 5.07, que facilita una lectura de las interacciones entre áreas y bloques curriculares, en este caso, aplicando la intermediación de los nodos (Vector/Centrality/Betweenness), lo que permite detectar los vectores principales de la red.

Como puede observarse en el grafo, todos los bloques de contenidos aparecen mencionados por el alumnado, pero con diferentes densidades. Vemos cómo son tres los nodos que fundamentalmente se destacan en la red: el A2.B3 (Cultura y vida en sociedad) y el A2.B2 (Acercamiento a la naturaleza), ambos del área 2. "Conocimiento del entorno"; junto con al A1.B3 (La actividad y la vida cotidiana) que, sorprendentemente, es el que mayor volumen de conexiones presenta con el resto de los bloques y áreas. Este gráfico confirma los datos obtenidos y analizados, evidenciando un notable esfuerzo del alumnado por globalizar los contenidos curriculares, en contraposición con la infrarrepresentación de algunos bloques, como puede ser el bloque A1.B1 (El cuerpo y la propia imagen), el A1.B2 (Juego y movimiento), el A2.B1 (Medio físico: Elementos, relaciones y medidas) o el A3.B2 (Lenguaje audiovisual y tecnologías de la información y de la comunicación). 


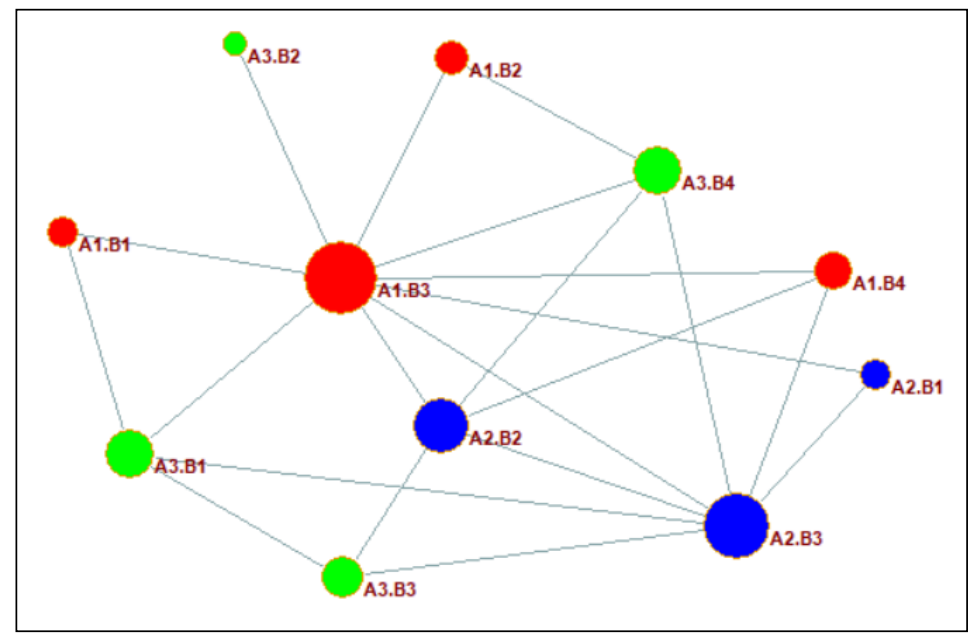

Gráfico 1. Representación relacional de los bloques y áreas del currículo de Educación Infantil, presentes en las producciones del alumnado ${ }^{\dagger}$

Desde un segundo nivel de análisis, respondemos al segundo objetivo de investigación planteado, acerca de si el alumnado es capaz de reconocer y delimitar los contenidos sociales del currículo de Educación Infantil. En esta fase de resultados vamos a analizar los tópicos o temas que el alumnado escogió, a fin de comprobar en qué medida conocen el currículo social de esta etapa y sus principales temáticas. Tras un intenso proceso de codificación, se establecieron 21 categorías (con 283 nodos) que se recogen en el Gráfico 2. A través de esta categorización ha sido posible realizar un análisis relacional de los nodos, con objeto de conocer mejor los temas con los que el profesorado en formación inicial vincula las Ciencias Sociales.

En primer lugar, es necesario evidenciar cómo de las 21 categorías codificadas, según su temática, algo más de la mitad (11) corresponden a contenidos de Ciencias Sociales, vinculados al bloque 3 "Cultura y vida en sociedad". Sin embargo, es llamativo que junto a ellos se incluyan 7 tópicos propios del bloque 1 y 2 del área de "Conocimiento del entorno", relacionados con el medio físico (A2.B1) ("reciclaje" o "ABN"), y con el acercamiento a la naturaleza (A2.B2) (“alimentación”, “astronomía”, "naturaleza”, "seres vivos” o "paisaje”). Asimismo, existen 3 tópicos más que hemos considerado transversales por su carácter no disciplinar: "currículo", "metodología” y "salidas didácticas".

\footnotetext{
${ }^{\dagger}$ Codificación definida en Tabla 3. En color rojo, bloques del área 1: A1.B1: Bloque 1. El cuerpo y la propia imagen; A1.B2: Bloque 2. Juego y movimiento; A1.B3: Bloque 3. La actividad y la vida cotidiana; A1.B4: Bloque 4. El cuidado personal y la salud. En color azul, bloques del área 2: A2.B1: Bloque 1. Medio físico: elementos, relaciones y medida; A2.B2: Bloque 2. Acercamiento a la naturaleza; A2.B3: Bloque 3. Cultura y vida en sociedad. En color verde, bloques del área 3: A3.B1: Bloque 1. Lenguaje verbal; A3.B2: Bloque 2. Lenguaje audiovisual y tecnologías de la información y la comunicación; A3.B3: Bloque 3. Lenguaje artístico; A3.B4: Bloque 4. Lenguaje corporal.
} 


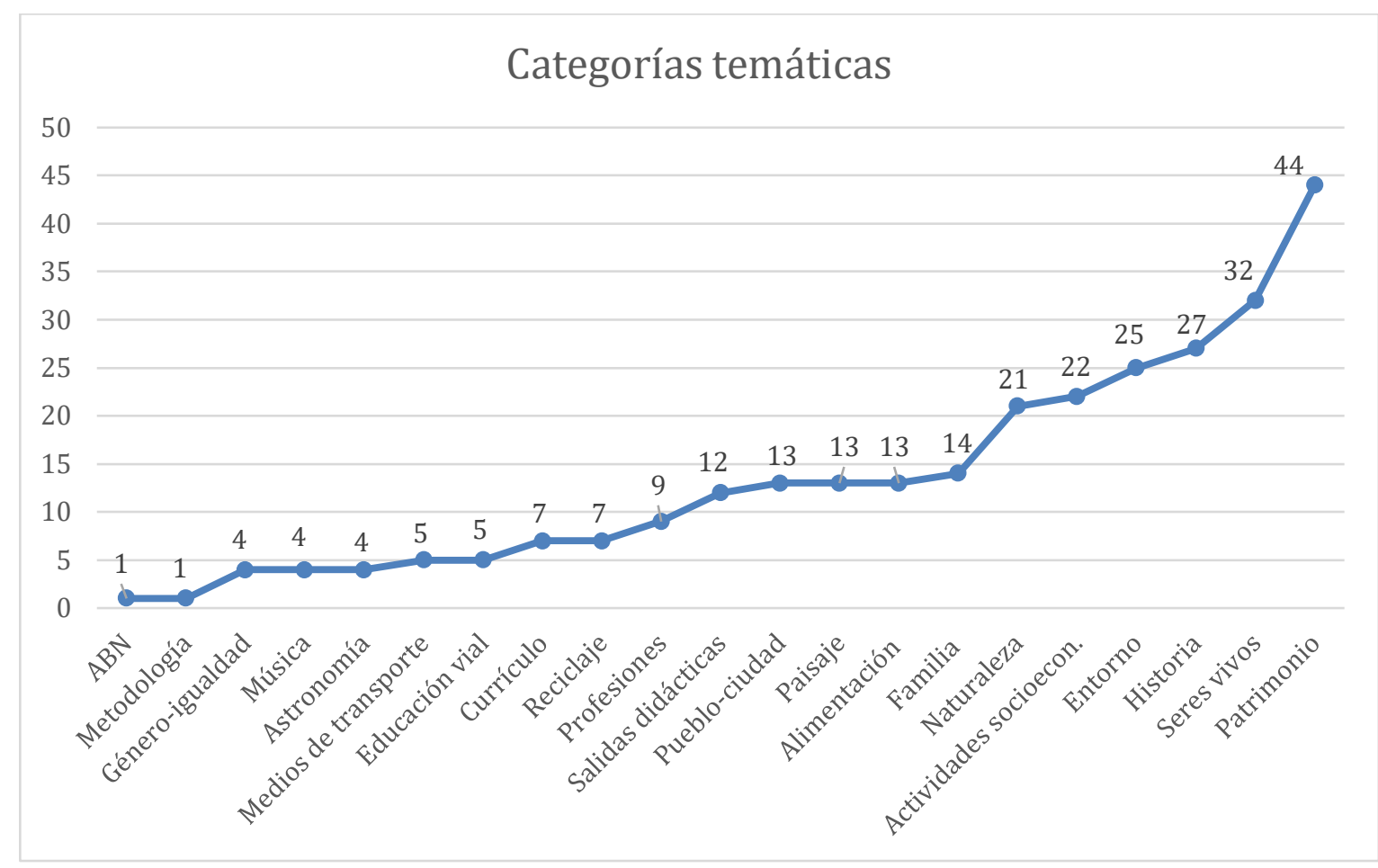

Gráfico 2. Categorías temáticas establecidas en la codificación y presencialidad de las mismas

De los contenidos vinculados con Ciencias Sociales, vemos cómo destacan con un mayor número de presencialidad las categorías: "patrimonio" (44), "historia” (27), "entorno" (25), “actividades socioeconómicas" (22) y “familia” (14), temas que guardan una íntima relación con el contenido social previsto para la etapa de infantil. En cuanto al término "patrimonio", las referencias son mayoritariamente del patrimonio conocido o cercano, aunque también se mencionan algunos elementos patrimoniales más alejados en el espacio, de amplia relevancia. En cuanto a las categorías de "historia" y "entorno", cuentan respectivamente con 27 y 25 menciones, por su íntima relación con los conceptos de tiempo y espacio, los cuales, pese a no estar explícitamente mencionados en el currículo, forman la columna vertebral del conocimiento social en infantil. Por su parte, el nodo "actividades socioeconómicas" tiene igualmente una frecuencia importante (22 nodos), ya que el alumnado ha considerado que pueden servir para introducir al niño/a tanto en el conocimiento de su sociedad, como en aspectos vinculados con la cultura, el patrimonio o las nociones temporales, sobre todo, si hablamos de actividades socioeconómicas del pasado. Cabe destacar por último la "familia", otra de las categorías más repetidas -aunque con menor frecuencia- por su presencia en el currículo y por su importancia, junto a la escuela, como primer agente de socialización.

Para finalizar con los resultados, y unificando ambos niveles de análisis, hemos creído conveniente representar visualmente en el Gráfico 3, las interacciones que se producen entre los nodos de las 21 categorías temáticas y los nodos de los 11 bloques de contenidos del currículo (554 interacciones). En este gráfico pueden observarse, en el centro de la imagen, los bloques que poseen un mayor número de relaciones (A1.B3, A2.B3 y A2.B2), lo que explica su mayor tamaño. Estas tres categorías coinciden con aquellos bloques con los que el alumnado ha conectado en mayor medida los contenidos sociales (Gráfico 1). 



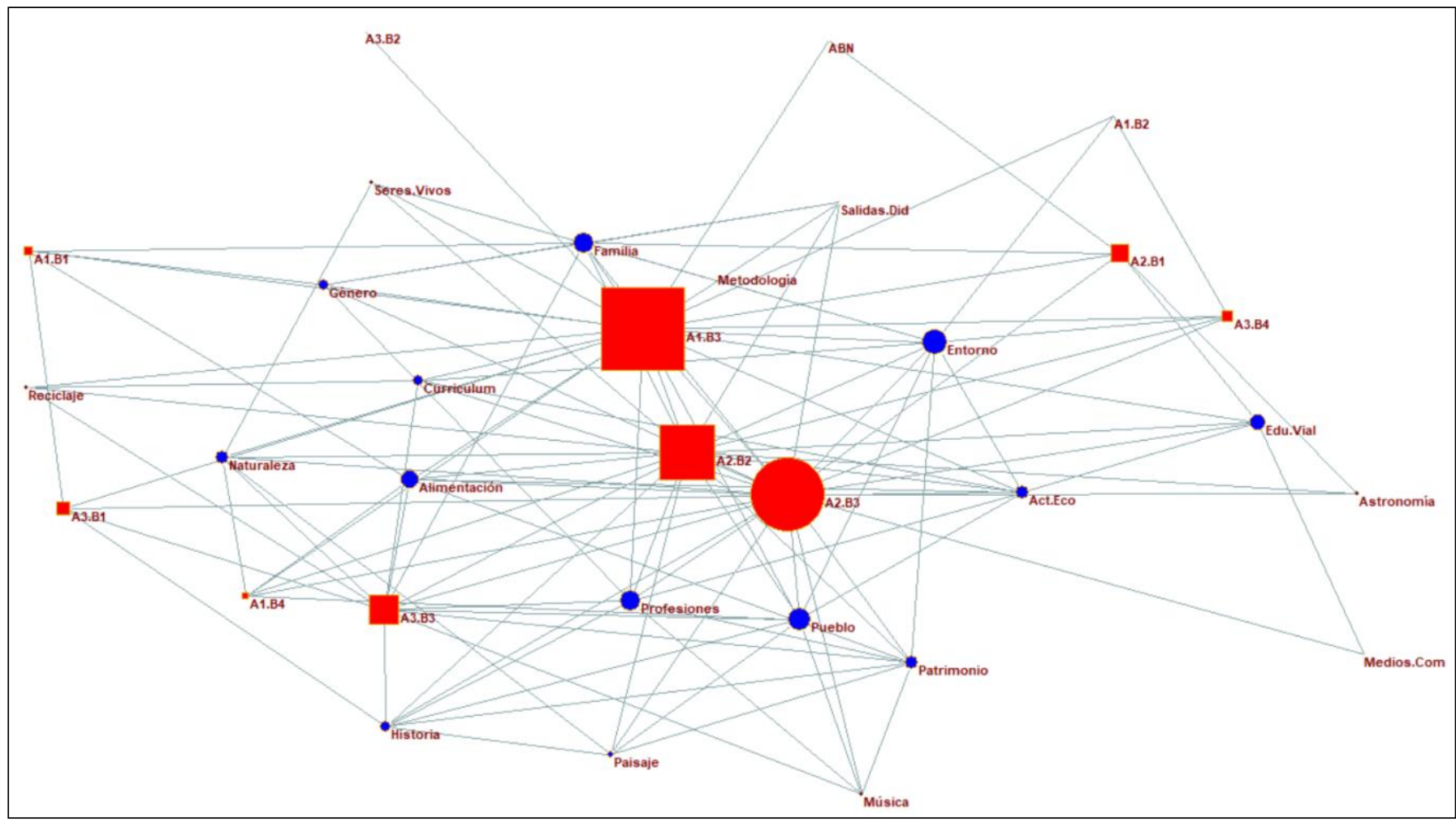

Gráfico 3. Conexiones entre bloques curriculares y temas categorizados en las producciones del alumnado

\begin{abstract}
${ }^{3}$ En color rojo se representan los bloques del currículo de Educación Infantil, siendo el círculo central (A2.B3) el bloque de "Cultura y vida en sociedad", donde se recogen los contenidos sociales. Los nodos circulares en azul son las temáticas que han sido categorizadas en las producciones del alumnado. Su mayor o menor tamaño depende del volumen de conexiones que existen entre estos nodos y los bloques del currículo. Existe un nodo, "Metodología", que aparece suelto por no
\end{abstract} tener conexión con ninguna área o bloque curricular. 
Un primer análisis del resultado visual obtenido nos permite constatar la notable densidad relacional de la red en torno a sus principales vectores (A1.B3, A2.B3 y A2.B2), lo que revela una destacada relación de los temas de elección libre (categorías temáticas) con los bloques y áreas curriculares. Conforme se va extendiendo el gráfico, vemos cómo la densidad de estas redes va disminuyendo, lo que refleja una menor interacción entre los nodos y, en consecuencia, una menor mención por parte del alumnado.

Las conexiones e interacciones representadas entre unos nodos y otros desvelan, en líneas generales, un nivel aceptable tanto de conocimiento del currículo social, como de conexiones entre aprendizajes, lo que denota una cierta capacidad en el alumnado para globalizar e integrar contenidos curriculares de distintas áreas. No obstante, se precisa de mejoras tanto en la conexión entre contenidos, como en la delimitación del conocimiento social curricular previsto para la Educación Infantil.

\section{Discusión y conclusiones}

Como reflejan los resultados expuestos, el profesorado en formación inicial tiene ciertas dificultades para ejercer el principio de globalidad y conectar los aprendizajes sociales con contenidos de otros bloques y áreas del currículo, siendo mayoritarios los vínculos que establece con contenidos de su propia área (Conocimiento del entorno), principalmente, con aquellos vinculados al medio natural. Fuera de esta área, de forma excepcional, tiende a la conexión con el área 1 de "Conocimiento de sí mismo y autonomía personal", especialmente con su bloque 3 "La actividad y la vida cotidiana", sin embargo, las referencias a sus bloques 1 y 2 , sobre el cuerpo y la propia e imagen, y el juego y el movimiento, son prácticamente nulos.

Esta circunstancia es probable que tenga su origen en las concepciones previas con que cuentan los futuros maestros sobre los usos y finalidades de las Ciencias Sociales. De acuerdo con Parra Monserrat (2019) y Morales y Santana (2019), es fundamental conocer las concepciones previas del alumnado y sus recuerdos escolares para poder incidir en ellos, deconstruirlos y transformarlos en nuevos modelos. Según estos autores, las experiencias con el conocimiento social no suelen ser valoradas positivamente, ya que se vinculan mayoritariamente a procesos de enseñanza y aprendizaje tradicionales y memorísticos, que provocan rechazo y dificultan la adaptación a las nuevas necesidades educativas. En lo que respecta a nuestra investigación, es posible que las concepciones del alumnado hacia las Ciencias Sociales les lleve a disociarlo del juego, de lo lúdico, y de la importancia que ejerce tanto en el desarrollo personal, como en la construcción de la propia imagen, adoptando una visión errónea de las finalidades y utilidades que puede tener el contenido social en Educación Infantil.

Las referencias al área de "Lenguajes", han sido igualmente anecdóticas, sobre todo en lo que respecta al bloque 2, "Lenguaje audiovisual y tecnologías de la información y de la comunicación". En este caso, los resultados denotan una falta de integración y conexión entre las nuevas tecnologías y los contenidos sociales, lo que nos lleva irremediablemente a cuestionarnos sobre el modo en que se han incorporado las nuevas tecnologías a las aulas universitarias, y la formación que está recibiendo el futuro profesorado al respecto. Según recientes investigaciones, todo parece apuntar que, pese al entusiasmo mostrado por los 
futuros maestros a emplear en sus aulas las nuevas tecnologías, lo cierto es que carecen de la formación necesaria para ello, lo que dificulta su adecuada integración en los procesos de enseñanza y aprendizaje (Cózar y Roblizo, 2014; Roblizo, Sánchez y Cózar, 2016; Rodríguez, López y Martín, 2017). En este sentido, se evidencia la necesidad de reforzar la formación inicial de maestros en competencias digitales si queremos capacitarles para que puedan desarrollar a su vez esta competencia en su alumnado (Carrera y Coiduras, 2012).

Por otra parte, creemos que la ausencia de conexiones entre el contenido social y los lenguajes audiovisuales y las nuevas tecnologías puede tener su origen también en los recuerdos escolares que vinculan Ciencias Sociales con estrategias didácticas rutinarias y poco motivadoras. Se podría inferir, por tanto, una tendencia a relacionar los contenidos sociales con elementos del pasado (patrimonio, monumentos, folclore, tradiciones, festividades...), sin contemplar en las tecnologías emergentes una alternativa innovadora para su enseñanza.

En cuanto al grado de conocimiento del currículo social de Educación Infantil que posee el alumnado podemos afirmar que es superficial, pues pese a tener claras algunas temáticas, confunde contenidos de los distintos ámbitos de conocimiento. La presencia de nodos o categorías no específicamente sociales, enmarcadas en los parámetros, principalmente, de las Ciencias Naturales, evidencia dificultades para establecer qué contenidos son propios de cada uno de los bloques del currículo.

Si nos centramos en las categorías más repetidas como temas sociales, destacan "patrimonio" "historia" y "entorno". En el primer caso, la amplia repetición del tema patrimonio se debe a la relevancia que hemos otorgado a emplearlo como recurso en la Educación Infantil, no solo para trabajar el espacio y el tiempo, sino otras áreas del currículo. Conscientes de su amplio potencial, se convierte en una herramienta clave para la globalización de aprendizajes y la aproximación del niño/a al entorno, en todas sus dimensiones (Miralles y Rivero, 2012; Escribano-Miralles, 2015; Aranda, 2016: 321). Sin formar parte, de modo explícito, de los contenidos propios de Educación Infantil, se valora positivamente las referencias que hace el alumnado a los bienes patrimoniales, tanto materiales como inmateriales, para trabajar los contenidos de esta etapa. En cuanto a la temática "historia", es significativo que, pese a no existir mención directa a contenidos históricos en la legislación, los alumnos y alumnas consideran que la mejor manera de identificar cambios en el modo de vida y las costumbres es precisamente abordando el paso del tiempo y las transformaciones que este comporta. Por su parte, la categoría "entorno" era previsible que se reflejara con una alta frecuencia, ya no solo por su amplia presencia en el currículo, sino también por la importancia que se le otorga como concepto vinculado al espacio en la Educación Infantil.

Junto a las categorías señaladas, es preciso detenerse además en las temáticas que no han estado presentes en las producciones del alumnado, pero que, sin embargo, están recogidas en el currículo. Estos temas son: la importancia de las relaciones sociales fuera del ámbito familiar (amistad, compañerismo, por ejemplo); el aprendizaje de conductas, comportamientos, normas o valores; derechos y deberes propios de una ciudadanía democrática; el conflicto y estrategias para la resolución de problemas; y, nuevamente, las nuevas tecnologías. Reflexionar sobre estas ausencias es fundamental porque nos da pistas 
acerca de qué contenidos no se han trabajado adecuadamente en el contexto de nuestras clases.

Dando respuesta a los objetivos de la investigación planteados más arriba, concluimos que existen ciertas lagunas en las competencias curriculares específicas adquiridas por el alumnado del Grado en Educación Infantil, tanto para concretar el conocimiento social curricular, como para diseñar situaciones de aprendizaje globalizadoras, resultados que, por otra parte, difieren con los aportados por González, González, Martínez y Pérez (2014), quienes concluyeron en su estudio que las competencias mejor valoradas por los estudiantes del Grado en Educación Infantil, en lo que a su adquisición se refiere, eran precisamente aquellas relacionadas con el conocimiento de contenidos curriculares y el principio globalizador. Aunque las muestras de ambos trabajos son de carácter limitado, no extensibles o extrapolables a otros contextos, no deja de ser significativo el contraste de resultados. Creemos que es posible que los estudiantes se sientan más seguros de haber alcanzado estas competencias -al menos, en apariencia-, porque desde todas las asignaturas del Grado se incide bastante en estos aprendizajes por ser de carácter técnico y estar vinculados al saber docente. Sin embargo, al menos desde nuestra investigación, todo parece indicar que, cuando se solicita al alumnado transcender del saber, al saber hacer, los resultados son otros.

Resta seguir trabajando para que el desarrollo de competencias profesionales sea el adecuado y para que estas puedan ser conveniente evaluadas. En este sentido, consideramos que este trabajo nos ha servido para establecer líneas de actuación que permitan subsanar las debilidades detectadas, mejorar nuestras prácticas docentes y optimizar la calidad formativa de nuestro alumnado.

\section{Referencias}

Alsina, A. (2013). Un modelo realista para el desarrollo profesional en la formación inicial de maestros de educación infantil. Revista Electrónica Interuniversitaria de Formación del Profesorado, 16(2), 27-37. DOI: http://dx.doi.org/10.6018/reifop.16.2.180761

Aranda Hernando, A. M. (2016). Didáctica de las ciencias sociales en Educación Infantil. Madrid: Editorial Síntesis.

Bisquerra Alzina, R. (2004). Metodología de la investigación educativa. Madrid: La Muralla.

Blasco Mira, J. E. y Mengual Andrés, S. (2010). Análisis de datos cualitativos asistido por ordenador en Ciencias de la Educación. En R. Roig Vila \& M. Fiorucci (Eds.), Claves para la investigación en innovación y calidad educativas. La integración de las Tecnologías de la Información y la Comunicación y la Interculturalidad en las aulas (pp. 71-84). Alcoy: Editorial Marfil.

Calvani, A. (1988). Il bambino, il tempo, la storia. Florencia: La Nuova Italia.

Carrera, F. X. \& Coiduras, J. L. (2012). Identificación de la competencia digital del profesorado universitario: un estudio exploratorio en el campo de las Ciencias Sociales. Revista de docencia Universitaria (REDU), 10(2), 273-298.

Cohen, L. y Manion, L. (2002). Métodos de investigación educativa Madrid: La Muralla. 
Comes, P. (2002). La interpretación de las imágenes espaciales. En P. Martínez Gracia, I. González Gallego y J. Anadón Benedicto (aut.), La geografía y la historia, elementos del medio (pp. 209-244). Madrid: Ministerio de Educación, Cultura y Deporte.

Cooper, H. (2002). Didáctica de la historia en la educación infantil y primaria. Madrid: Morata.

Cózar Gutiérrez, R. y Roblizo Colmenero, M. J. (2014). La competencia digital en la formación de los futuros maestros: percepciones de los alumnos de los Grados de Maestro de la Facultad de Educación de Albacete. RELATEC, Revista Latinoamericana de Tecnología Educativa, 13(2), 119-133. DOI: 10.17398/1695288X.13.2.119.

Cuenca López, J. M. (2008). La enseñanza y el aprendizaje de las ciencias sociales en Educación Infantil. En R. M. Ávila, M. A. Cruz y M. C. Díez (eds.)., Didáctica de las Ciencias Sociales, currículo escolar y formación del profesorado (pp. 289-312). Jaén: Universidad de Jaén.

Cuenca López, J. M. y Domínguez Domínguez, C. (2000). Un planteamiento socio-histórico para la educación infantil. Íber. Didáctica de las ciencias sociales, geografía e historia, 23, 113-124.

Escribano-Miralles, A. (2015). Utilización del patrimonio para el aprendizaje de la historia en educación infantil. Experiencia educativa “¡Esta clase es un Museo!”, Pulso. Revista de Educación, 38, 179-206.

Gil Flores, J. y Perera Rodríguez, V. (2001). Análisis informatizado de datos cualitativos. Sevilla: Kronos.

González Lorente, C., González Morga, N., Martínez Juárez, M. y Pérez Cuso, J. (2014). Desarrollo y relevancia de las competencias específicas en el Grado de Educación Infantil desde la percepción de los estudiantes de último curso. En J. J. Maquilón Sánchez y N. Orcajada Sánchez (eds.), Investigación e innovación en formación del profesorado (pp. 107-119). Murcia: Editum.

Hannoun, H. (1977). El niño conquista el medio. Las actividades exploradoras en la escuela primaria. Buenos Aires: Kapelusz.

López Meneses, E., Domínguez Fernández, G., Álvarez Bonilla, F. J. y Jaén Martínez, A. (2011). Experiencia didáctica con estudiantes de posgrado sobre los roles del educador en la Sociedad del Conocimiento y la Comunicación con tecnologías 2.0. RELATEC, Revista Latinoamericana de Tecnología Educativa, 10(1), 49-58.

Miralles Martínez, P. y Molina Puche, S. (2011). Didáctica de las Ciencias Sociales para el área de Conocimiento del entorno. En M. P. Rivero Gracia (coord.), Didáctica de las Ciencias Sociales para Educación Infantil (pp. 89-110). Zaragoza: Mira Editores.

Miralles Martínez, P. y Rivero Gracia, M. P. (2012). Propuestas de innovación para la enseñanza de la historia en Educación Infantil. Revista Electrónica Interuniversitaria de formación del profesorado,15(1), 81-90.

Morales Hernández, A. J. y Santana Martín, D. (2019). Las representaciones de la Didáctica de las Ciencias Sociales en los futuros docentes de Educación Infantil. En D. Parra Monserrat y C. Fuertes Muñoz (Coords.), Reinterpretar la tradición, transformar las 
prácticas. Ciencias Sociales para una educación crítica (pp. 99-124). Valencia: Editorial Tirant lo Blanch.

Orden $\mathrm{ECl} / 3854 / 2007$, de 27 de diciembre, por la que se establecen los requisitos para la verificación de los títulos universitarios oficiales que habiliten para el ejercicio de la profesión de Maestro en Educación Infantil. BOE, número 312, publicado el sábado 29 de diciembre de 2007

Parra Monserrat, D. (2019). Representación de la Historia escolar y crítica del consenso del sentido común. En D. Parra Monserrat y C. Fuertes Muñoz (Coords.), Reinterpretar la tradición, transformar las prácticas. Ciencias Sociales para una educación crítica (pp. 73-98). Valencia: Editorial Tirant lo Blanch.

Piñuel Raigada, J. L. (2002). Epistemología, metodología y técnicas del análisis de contenido. Estudios de Sociolingüística, 3(1), 1-42.

Real Decreto, 1630/2006, de 29 de diciembre, por el que se establecen las enseñanzas mínimas del segundo ciclo de Educación Infantil. BOE, núm. 4, publicado el 4 de enero de 2007.

Rivero Gracia, M. P. y Gil Alejandre, J. (2011). Pensar y expresar el espacio en el aula de Infantil. En M. P. Rivero Gracia (coord.), Didáctica de las Ciencias Sociales para Educación Infantil (pp. 31-47). Zaragoza: Mira Editores.

Roblizo Colmenero, M., Sánchez Pérez, M. C. y Cózar Gutiérrez, R. (2015). El reto de la competencia digital en los futuros docentes de infantil, primaria y secundaria: los estudiantes de grado y máster de educación ante las TIC. Prisma social: revista de investigación social, 15, 254-295.

Rodríguez, M. R., López, A. y Martín, I. (2017). Percepciones de los estudiantes de Ciencias de Educación sobre las redes sociales como metodología didáctica. Píxel-Bit-Revista de Medios y Educación, 50, 77-93. DOI: 10.12795/pixelbit.2016.i50.05.

Rosales López, C. (2013). Competencias específicas curriculares que ha de adquirir el estudiante del título de grado de maestro. Profesorado. Revista de Currículum y Formación de Profesorado, 17(3), 73-90.

Tárraga-Mínguez, R., Sanz-Cervera, P., Pastor-Cerezuela, G. y Fernández-Andrés, M. I. (2017). Análisis de la autoeficacia percibida en el uso de las TIC de futuros maestros y maestras de Educación Infantil y Educación Primaria. Revista Electrónica Interuniversitaria de Formación del Profesorado, 20(3), 107-116. DOI: http://dx.doi.org/10.6018/reifop.20.3.263901

Tonda, M. E. (2001). La Didáctica de las Ciencias Sociales en la formación del profesorado de Educación Infantil. Alicante: Publicaciones de la Universidad de Alicante.

Trepat, C. A. (2011). El aprendizaje del tiempo en educación infantil. En M. P. Rivero Gracia (coord.), Didáctica de las Ciencias Sociales para Educación Infantil (pp. 49-63). Zaragoza: Mira Editores.

Valdemoros-San-Emeterio, M. A., Ponce-de-León, A. y Sanz-Arazuri, E. (2011). Fundamentos en el manejo del Nvivo 9 como herramienta al servicio de estudios cualitativos. Contextos Educativos, 14, 11-29. 